Open Access

\title{
Characterization of Patients with Difficult-to-Treat Acute Pain Following Total Knee Arthroplasty Using Multi-Modal Analgesia
}

\author{
Syed A. Azim ${ }^{1}$, Rebecca Sangster ${ }^{1}$, Christine Curcio ${ }^{1}$, Dominick Coleman ${ }^{1}$, Usman Shah $^{1}$, \\ Shaonan Zhang ${ }^{2}$, Ruth A. Reinsel, Peter S.A. Glass ${ }^{1}$, James Nicholson ${ }^{3}$ and Helene Benveniste*,
}

\author{
${ }^{I}$ Department of Anesthesiology, Stony Brook Medicine, Stony Brook, NY, USA; ${ }^{2}$ Department of Applied Mathematics \\ and Statistics, Stony Brook University, NY, USA; ${ }^{3}$ Department of Orthopaedics, Stony Brook Medicine, Stony Brook, NY, \\ USA
}

\begin{abstract}
Objective: To determine the efficacy of post-operative pain control over the first 24 hours in patients undergoing unilateral total knee arthroplasty (TKA) using a multi-modal analgesia protocol including femoral nerve catheters (FNC). Materials and Methods: 119 patients who underwent unilateral TKA between 2009 and 2010 under regional anesthesia with FNC were studied. Electronic charts were reviewed for numerical rating scale (NRS) pain scores at baseline, from the time the patients entered the post-operative care unit and every 4 hours thereafter until FNC was discontinued at 24 hours post-surgery. Opioid usage was also recorded during the same time period. Results: Analysis of average NRS pain scores from all patients demonstrated that $69 \%$ had NRS pain scores $\leq 5$ ('low pain' (LP) group) and $31 \%$ had NRS pain scores $\geq 6$ ('high pain' (HP) group). Time analysis showed that HP patients' high pain scores persisted for 24 hours post-surgery and they were characterized by being younger when compared to the LP patients.The majority of HP patients were female. Further analysis demonstrated that the average body mass index (BMI) of the female HP patients was significantly greater than females with LP. Conclusions: In spite of a diverse multi-modal analgesia protocol designed for TKA surgery, $31 \%$ of our patients had 'difficult-to-treat' pain (NRS pain scores $\geq 6$ ) for 24hours postsurgery. Our analysis implicates age, pre-operative pain scores, female gender and obesity as potential risk factors for experiencing insufficient pain control with the currently evaluated multimodal pain protocol post-TKA surgery.
\end{abstract}

Keywords: Acute pain, female, multi-modal analgesia, obesity, post-operative pain, total knee arthroplasty.

\section{INTRODUCTION}

Clinical data confirm that total knee arthroplasty (TKA) is a painful procedure, and numerical pain rating scale (NRS) scores (with 0 signifying no pain and 10 the worst pain) over the first 24 hours post-surgery can be as high as 5-7 depending on the anesthesia and post-surgical pain management techniques used [1-5]. Health related websites have been created to educate prospective patients about what to expect in regard to pain following TKA surgery. Within these websites statements are posted such as "pain after knee surgery is quite variable and not entirely predictable" [6] or "recovering from knee replacement surgery can be painful" [7].

In most clinical anesthesia practices standardized opioidsparing, multimodal analgesia protocols [8] that include regional anesthesia techniques (e.g. femoral nerve catheters [9-12]), non-opioid analgesics (e.g. Cox-2 inhibitors [5], pregabalin [13]), intrathecal [14-16] and systemic opioids have been shown to be relatively effective in controlling pain for orthopedic procedures such as TKA. In line with these practice guidelines, at our institution TKA patients are offered a multimodal peri-operative analgesia regimen

*Address correspondence to this author at the Department of Anesthesiology, Stony Brook Medicine, Stony Brook, NY, USA; Tel: (631) 444-2975; Fax: (631) 444-2907;

E-mail: Helene.benveniste@stonybrookmedicine.edu comprising non-opioid analgesics, opioids and spinal anesthesia in combination with femoral nerve blockade plus indwelling femoral nerve catheter (FNC) [17]. Our clinical goal has been to provide adequate acute post-operative pain control for our TKA patients and based on the literature defined as average NRS pain scores $\leq 5$, using an11-point verbal NRS scale over the first 24 hours post-surgery. To validate our clinical expectations in regard to analgesia management in our patients we executed a retrospective study to characterize pain profiles over the first 24 hours in 119 patients undergoing unilateral TKA.

\section{MATERIALS AND METHODOLOGY}

Approval for this retrospective study was received from the local institutional review board (IRB), and consent from the patients to access their electronic medical records was waived by the IRB. Electronic medical charts for 119 patients who underwent elective unilateral TKA during 2009-2010 were reviewed. Patients were excluded from the study collection review if they had undergone TKA under general anesthesia, if they were on opioid therapy at the time of surgery or if there was documented failure of the FNC during the first 24 hours post-surgery. Data collection forms included demographic data, past medical and surgical history, peri-operative vital signs, pre-surgical baseline NRS pain scores, NRS pain scores from the post-anesthesia care unit (PACU), and those reported by the ward nurse every 4 hours until the FNC was discontinued by the acute pain team 
Table 1. Multi-Modal Anesthesia Regimen for TKA Patients

\begin{tabular}{|l|l|l|l|l|}
\hline $\begin{array}{l}\text { Technique \& Route of } \\
\text { administration }\end{array}$ & Medications & Dose & Start & End \\
\hline \hline Oral & Celebrex & $200 \mathrm{mg} \mathrm{q} 12 \mathrm{hrs}$ & Morning of Surgery & 48 hrs post-surgery \\
\hline Oral & $\begin{array}{l}\text { Oxycontin } \\
<70 \text { years of age }\end{array}$ & $20 \mathrm{mg} \mathrm{q} 12 \mathrm{hrs}$ & Morning of Surgery & 1 week post operatively \\
\hline Oral & $\begin{array}{l}\text { Oxycontin } \\
\geq 70 \text { years of age }\end{array}$ & $10 \mathrm{mg} \mathrm{q} 12 \mathrm{hrs}$ & Morning of Surgery post operatively \\
\hline Spinal anesthesia & $\begin{array}{l}0.75 \% \text { hyperbaric } \\
\text { bupivacaine }\end{array}$ & $10-15 \mathrm{mg}$ & Intra-operatively & N/A \\
\hline Femoral Nerve Catheter (FNC) & $\begin{array}{l}\text { Ropivacaine } \\
\text { Ropivacaine) pre- } \\
\text { operatively }\end{array}$ & $\begin{array}{l}\text { Upon recovery of motor function: } \\
\text { atusion via FNC: } 0.2 \% \text { Ropivacain }\end{array}$ & at 24 hrs post-surgery \\
\hline Oral & Pregabalin & 75 mg q $12 \mathrm{hrs}$ & Evening on day of surgery & 48 hrs post-surgery \\
\hline
\end{tabular}

at 24 hours post-surgery. In addition, FNC removal time (including total local anesthetic infusion time via the FNC) and opioid usage was recorded (defined as the amount of morphine equivalents recorded in milligrams administered to the patient during the first $24 \mathrm{hrs}$ ).

\section{Multi-Modal Analgesia TKA Protocol}

The multi-modal anesthesia regimen used for the TKA patients comprised several different medications, anesthesia techniques and timing of medication administration as shown in Table 1. Celecoxib (Celebrex ${ }^{\circledR}$ ) and oxycontin were started on the morning of surgery (Table 1) and for surgical anesthesia all patients received lumbar spinal anesthesia with hyperbaric $0.75 \%$ bupivacaine in addition to a femoral nerve block with a bolus of $40 \mathrm{cc}$ of $0.5 \%$ ropivacaine administered via an indwelling FNC placed using ultrasound and electrical nerve stimulation guidance. Intra-operatively, all patients were sedated with intravenous propofol only. Post-operatively, the patients were transferred for recovery to the PACU and upon return of their lower extremity motor function (defined as the ability to move feet; lift and bend the non-operated leg) $0.2 \%$ ropivacaine at an infusion rate of $10 \mathrm{cc} / \mathrm{hr}$ was started via the FNC. Finally, after the patients were transferred from the PACU to the orthopedic floor they received $75 \mathrm{mg}$ Pregabalin (Lyrica ${ }^{\circledR}$ ) orally in the evening (Table 1). At our institution, a dedicated acute pain management team led by anesthesiologists manages patients' pain post-operatively. For breakthrough pain the patients were given oxycodone 5$10 \mathrm{mg}$ orally every 4 hours as needed. On the floor the patients typically start rehabilitation on the first postoperative day via a continuous passive motion machine (CPM) for 2 hour blocks up to 4 hours during the first 24 hours.

\section{Data and Statistical Analysis}

All data from the case report forms were transcribed onto electronic spreadsheets and subsequently analyzed using SAS statistical software (v. 9.2, SAS Institute, NC). As our clinical intention was to maintain a pain score at $\leq 5$ we divided patients into a low pain group (LP) if the average NRS pain score over 24 hours was $\leq 5$ or a high pain group
(HP) if the average pain was $\geq 6$. These two groups were then compared in regard to differences in NRS pain scores over time using repeated measures analysis of variance (ANOVA). To characterize potential underlying factors that distinguished LP from HP patients we performed the following analyses: a) a chi-square test on gender, b) 2-sided independent t-test on age, BMI, baseline NRS pain scores and duration of surgery, and c) logistic regression on 24 hour average NRS pain scores from HP and LP patients with gender, age, BMI and baseline NRS pain scores as predictors. Stepwise selection at a significance level of 0.05 was applied to determine the best model. Differences in comorbidity between the two groups were tested using the Fisher's exact test with post-hoc corrections for multiple comparisons. Opioid consumption between the two groups was compared using a one-way analysis of covariates (ANCOVA) to compare opioid consumption between the two groups with age as a covariate.

\section{Results}

Of the 119 patients who underwent TKA during the predetermined time period, a total of 106 electronic patient charts were reviewed (13 patients were excluded due to documented opioid usage prior to surgery).

\section{Post-Operative Pain Profiles Over 24 Hours}

We first calculated the average NRS pain scores for each TKA patient from the time the patients were transferred to the orthopedic ward by averaging NRS pain scores reported via the electronic records at 4, 8, 12, 16, 20 and 24 hours. The frequency distributions of the average NRS pain scores from all patients are shown in Fig. (1) and demonstrate that $69 \%$ of the patients had average NRS pain scores $\leq 5$ and $31 \%$ had average NRS pain scores $\geq 6$. Patients with NRS pain scores $\leq 5$ were assigned to the 'low pain' (LP) group and those with NRS pain scores $\geq 6$ were assigned to the 'high pain' (HP) group. Fig. (2) shows the time-course of NRS pain scores in the LP and HP groups measured from the time of PACU discharge and every 4 hours over 24 hours and demonstrates that NRS pain scores remained elevated in the HP group over the entire time period, in contrast to the LP group which had adequate pain control $(\leq 5)$ according to 


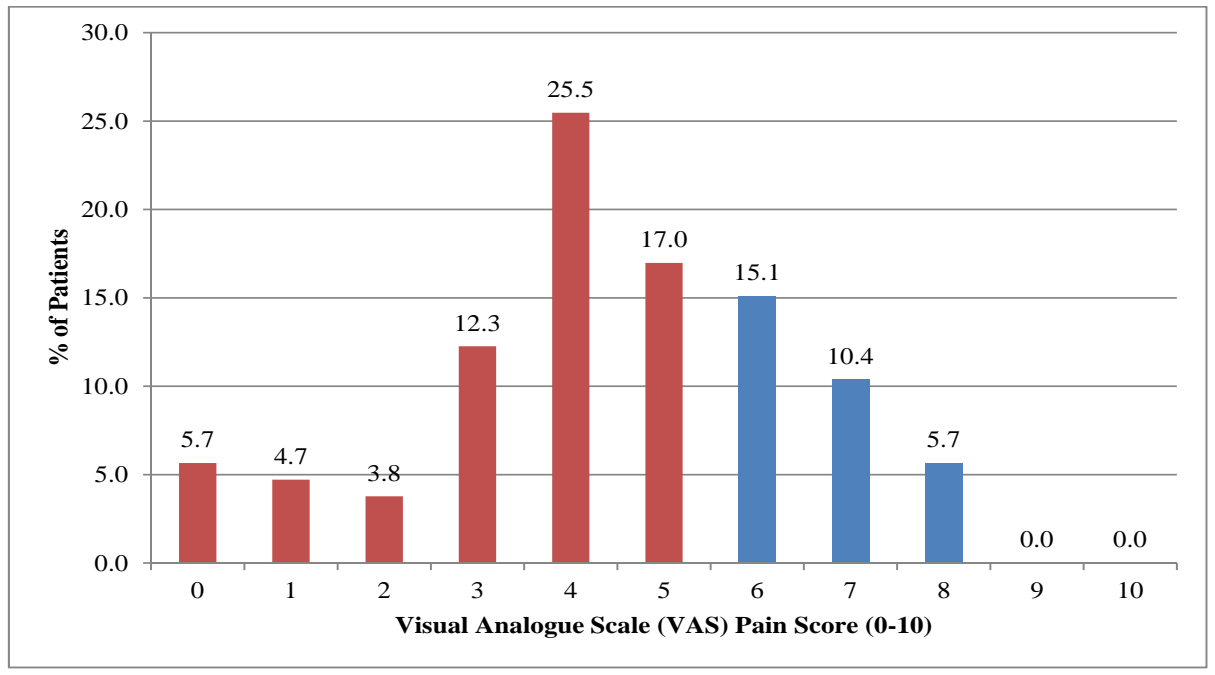

Fig. (1). Frequency Distributions of Average VAS Pain Scores in TKA Patients over first 24 hrs post-surgery.

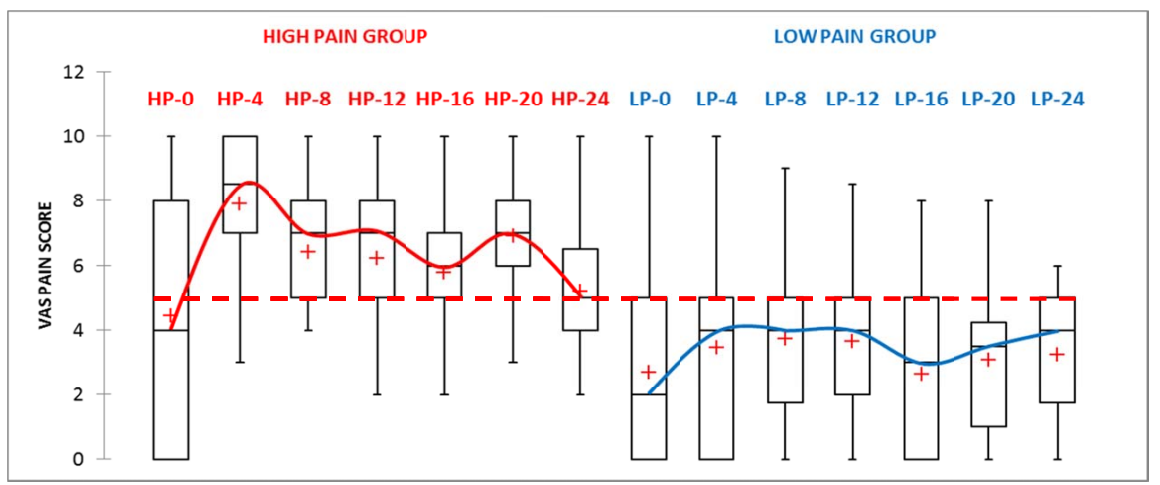

Fig. (2). Time-course of NRS pain scores in the LP and HP groups measured from the time of PACU discharge and every 4 hours over 24 hours.

clinical expectation. Changes in pain scores within and between LP and HP groups over time were found to be significant. Specifically, the change in pain over time was different between the two groups (Group * Time interaction effect, $p<0.0001$ ); and post-hoc analysis revealed that except for the time at PACU discharge, median NRS pain scores in the HP group were significantly different from the LP group at 4, 8, 16 and 20 hours post-operatively. Importantly, data analysis in regard to the FNC showed that in the high pain (HP) and low pain (LP) patient groups the average infusion time before the FNC was discontinued was $23.8 \pm 6.2 \mathrm{hrs}$ and $22.6 \pm 4.1 \mathrm{hrs}$, respectively (no significant difference, $\mathrm{p}$ value $=0.28$ ), suggesting that malfunction of the FNC did not contribute to differences in pain profiles between the two groups.

\section{Comparison of Demographics, Pre-operative NRS Pain Scores and Co-Morbidity Between LP and HP Groups}

Table 2 shows that age was significantly different between the two groups and younger age characterized patients in the HP group (LP: $69.7 \pm 11.1$ years versus HP $62.5 \pm 8.7$ years, $p=0.001)$ as well as higher baseline NRS pain scores. To further validate this finding we performed a logistic regression on 24 hour average NRS pain scores from the two groups with gender, age, BMI and baseline NRS pain scores as predictors. The regression coefficients for age and baseline NRS pain scores were -0.063 and 1.14 , respectively, and both were significant $(p=0.01$ for age and $p=0.04$ for baseline NRS pain). Explicitly, the model predicted that for patients 40 years or older the log odds ratio of getting a NRS pain score of 6 or greater for a given patient would decrease with increasing age $(6.1 \% /$ year) and increase with higher pre-surgical NRS pain scores. In regard to comorbidity, Table 2 shows that except for cardiac disease (stable coronary artery disease), which was significantly higher in the LP group, co-morbidity was matched between the two groups.

\section{Opioid Consumption Between LP and HP Groups}

As expected, the average opioid consumption as defined by morphine equivalents in $\mathrm{mg}$ over 24 hours postoperatively in the HP group was significantly higher when compared to the LP group $(28.7 \pm 20.8 \mathrm{mg}$ versus $17.5 \pm$ $15.1 \mathrm{mg}, \mathrm{p}=0.002$ ). However, opioid consumption over the first 24 hours in the two groups fluctuated widely as indicated by the high standard deviations (SD). After controlling for age, opioid consumption was still significantly different between the two groups (group effect: p-value $=0.016$ ) but the estimated SDs on opioid usage between the two groups were greatly reduced after age 
Table 2. Patient Characteristics

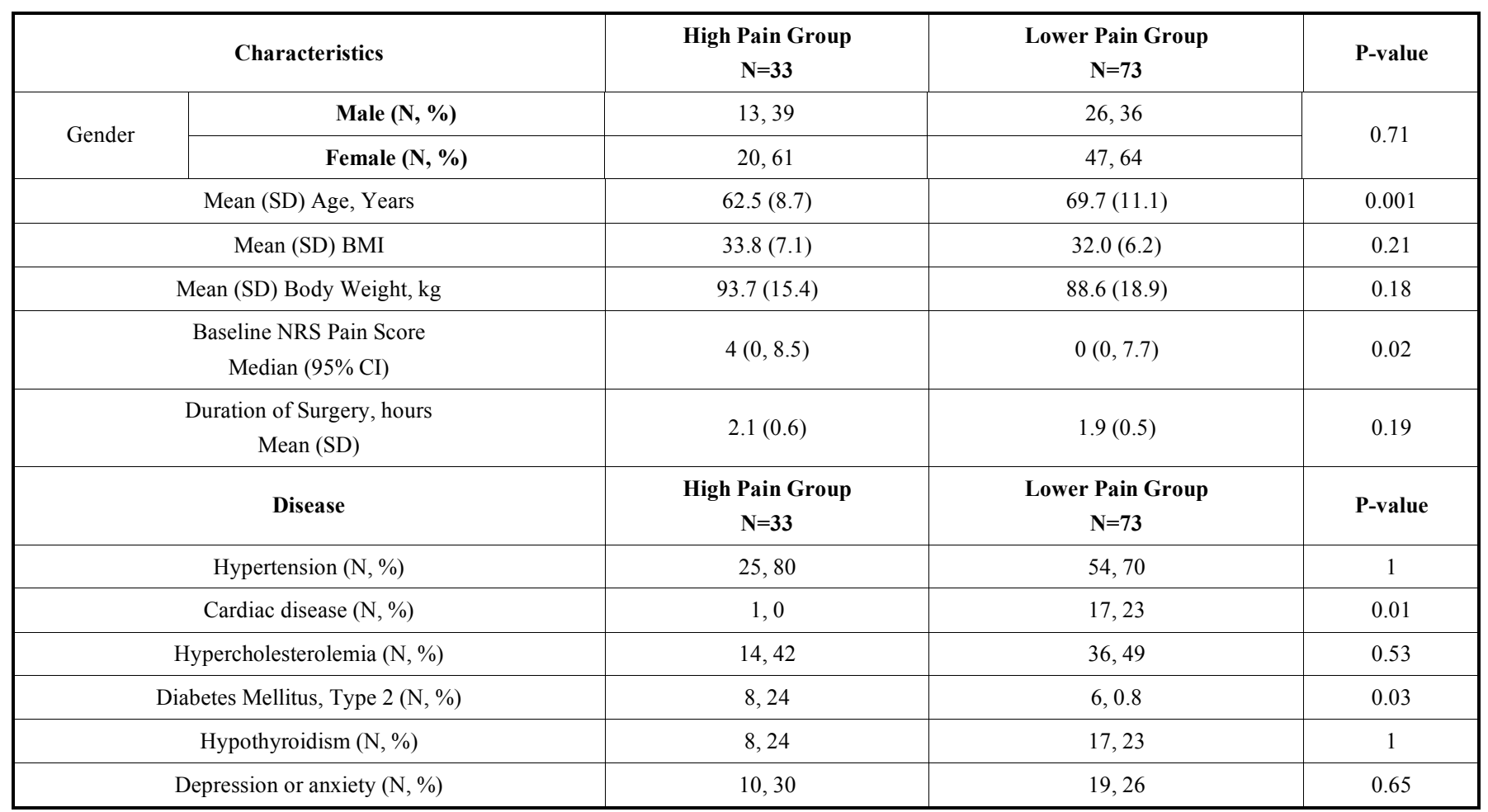

$\mathrm{N}=$ number of subjects.

adjustment, as shown in Fig. (3). However 'age' in itself was not a significant factor in regard to opioid consumption $(\mathrm{p}=0.08)$.

\section{Female TKA Patients}

Although gender distributions were matched between the LP and HP groups, we were interested in looking specifically at characteristics of female patients in the HP groups given that the majority of TKA patients are female (Table 2) and further that the post-operative pain has been reported to be more severe in female patients [18]. We therefore compared age, BMI and duration of surgery between the two groups using a 2-sided independent $t$-test while correcting for multiple comparisons. Interestingly, this analysis demonstrated that the BMI of the female patients in the HP group was $12 \%$ greater than the LP group (HP BMI: $37.3 \pm$ 6.4 versus LP BMI: $33.1 \pm 6.1, \mathrm{p}=0.01)$. Explicitly, $30 \%$ of the female patients in the HP group were characterized by a $\mathrm{BMI} \geq 40$; in comparison to only $17 \%$ in the LP group. This statistical difference also held true when body weights were compared (HP body weight: $96.6 \pm 15.5 \mathrm{~kg}$ versus LP body weight: $85.9 \pm 18.3, \mathrm{p}=0.03)$. In regard to co-morbidity, there were no significant differences between the LP and HP female patients. However, $46 \%$ of the obese patients in the HP group were treated for depression in comparison to only $21 \%$ in the LP group (not significant).

\section{DISCUSSION}

This retrospective analysis of 106 patients demonstrated that the multi-modal analgesia protocol we implemented for patients undergoing unilateral TKA did not provide uniform pain control over the first 24-hours post-operatively. Guided by the clinical literature we defined 'acceptable' average NRS pain scores to be $\leq 5$ over the first 24 hours, and the majority of TKA patients fell into this category. However, $31 \%$ of the TKA patients had NRS pain scores $\geq 6$ over the first 20 hours after PACU discharge in spite of the multimodal approach. Frequency analysis further demonstrated that as many as $16 \%$ of the patients had NRS scores $\geq 7$ which from a quality care point of view is unacceptable. Several studies have characterized acute post-operative pain in TKA patients treated with and without femoral nerve blockade and reported NRS pain scores similar to those reported here acutely after surgery [19-23]. For example; in the study by Wang and colleagues, the TKA patients' mean NRS pain score was reported to be 3 and 6 during rest and rehabilitation, respectively, 20-24 hours post-surgery [23]. As previously mentioned, at our institution, the TKA patients start rehabilitation on the first post-operative day via a continuous passive motion machine (CPM) for 2 hours during the first 24 hours, and the NRS pain scores reported by the ward nurse every 4 hours are incorporated in the CPM routine. However, we were unable to separate 'rest' from 'rehabilitation' NRS scores based on the retrospective data collection.

Further analysis of the post-surgical NRS scores demonstrated that patients in the HP group were defined as being slightly younger and having higher pre-surgical pain scores. Logistic regression analysis revealed that the odds of falling into the HP group were $6.6 \%$ lower for every year of 'aging' (assuming constant pre-surgical pain levels over time). This finding of younger age being a predictor of severe pain acutely after surgery is not novel and has been previously reported [24, 25]. In fact, Kalkman and coworkers [25] recently characterized predictors for 




Fig. (3). Opioid Usage in TKA patients with high and low NRS pain scores.

developing post-operative pain for a wide variety of surgical procedures including orthopedic procedures in a large series of surgical inpatients undergoing general anesthesia with either an inhalational anesthetic or propofol [25]. While there was no difference in the number of patients with severe pain between the two anesthesia techniques, younger age, female gender and high pre-operative pain scores were among the most significant predictors of 'presence of severe pain' postsurgery [25]. These classifiers have been confirmed in other studies as well [26, 27], although some studies do not find gender to be a significant factor in acute pain [28].

Our retrospective analysis also demonstrated that the majority of the female TKA patients with high, 'difficult-totreat' post-operative pain were characterized by being more obese (BMI $=37.7 \pm 6.1$ ), in contrast to the female patients in the low pain group who had an average BMI of $34.4 \pm 5.9$. To the best of our knowledge, there are no clinical studies in the literature demonstrating or suggesting that obese female patients are at higher risk for developing unmanageable, acute pain post-surgery. However, there is a growing body of literature on the role of fat itself and its many pathophysiological consequences. Previously, adipocytes were considered to be little more than storage cells for fat, but it is now clear that fat tissue functions as an active endocrine organ that produces many pro-inflammatory substances such as tumor necrosis factor alpha (TNF $\alpha$ ), interleukin 6 (LI-6) and leptin [29-33] which interact with many physiological processes. For example, leptin is produced by adipocytes and is a hormone that regulates food intake; leptin also can act as a cytokine and induce a heightened inflammatory state [34]. In this context it is also important to emphasize that plasma levels of leptin in obese subjects have been shown to correlate positively with BMI $[35,36]$ and that leptin levels are higher in females [37]. A current working hypothesis now associates obesity with a chronic inflammatory state, in other words, as individuals become obese and their adipocytes enlarge, adipose tissue undergoes molecular and cellular alterations that change body metabolism and induce a general inflammatory state $[38,39]$. Based on this evidence in the literature, it is possible that obesity in the female TKA patients may have played a role in regards to higher NRS pain scores postoperatively.

However, there are many other factors that potentially explain our findings and which could not be controlled for (e.g. oral rather than parenteral administration of opioids and other predisposing factors) in this retrospective study. Clearly, further investigation of the potential role of obesity and pre-operative inflammatory status on post-operative acute pain following TKA, including measurements of serum leptin levels, will be needed to provide further support and validation for our findings.

\section{CONFLICT OF INTEREST}

The authors confirm that this article content has no conflicts of interest.

\section{ACKNOWLEDGEMENTS}

The authors would like to thank Stony Brook Medicine's Department of Anesthesiology for providing the financial support for this study. Dr. Azim's participation in this research study was, in part, sponsored by the Stony Brook University School of Medicine Dean's Office Professional Development Program.

\section{REFERENCES}

[1] Clarke H, Pereira S, Kennedy D, et al. Gabapentin decreases morphine consumption and improves functional recovery following total knee arthroplasty. Pain Res Manag 2009; 14: 217-22.

[2] Frassanito L, Vergari A, Messina A, Pitoni S, Puglisi C, Chierichini A. Anaesthesia for total knee arthroplasty: efficacy of single-injection or continuous lumbar plexus associated with sciatic nerve blocks--a randomized controlled study. Eur Rev Med Pharmacol Sci 2009; 13: 375-82.

[3] Sanders JC, Gerstein N, Torgeson E, Abram S. Intrathecal baclofen for postoperative analgesia after total knee arthroplasty. J Clin Anesth 2009; 21: 486-92.

[4] Wang HJ, Zhang DZ, Li SZ. Comparing the analgesic efficacy of continuous femoral nerve blockade and continuous intravenous analgesia after total knee arthroplasty. Zhonghua Yi Xue Za Zhi 2010; 90: 2360-2.

[5] Buvanendran A, Kroin JS, Tuman KJ, et al. Effects of perioperative administration of a selective cyclooxygenase 2 inhibitor on 
pain management and recovery of function after knee replacement: a randomized controlled trial. JAMA 2003; 290: 2411-8.

[6] Activities After a Knee Replacement. Available at: http://orthoinfo.aa-os.org/to-pic.cfm?topic $=$ A00357, [Accessed: Sept. $4^{\text {th }}$, 2012].

[7] Recovering from Knee Replacement Surgery. Available at: http://www.everydayhealth.com/pain-management/knee-pain/jointreplacement.aspx, [Accessed: Sept. $4^{\text {th }}$ 2012].

[8] White PF, Kehlet H. Improving postoperative pain management: what are the unresolved issues? Anesthesiology 2010; 112: 220-5.

[9] Paauwe JJ, Thomassen BJ, Weterings J, van Rossum E, Ausems ME. Femoral nerve block using ropivacaine $0.025 \%, 0.05 \%$ and $0.1 \%$ : effects on the rehabilitation programme following total knee arthroplasty: a pilot study. Anaesthesia 2008; 63: 948-53.

[10] De Ruyter ML, Brueilly KE, Harrison BA, Greengrass RA, Putzke JD, Brodersen MP. A pilot study on continuous femoral perineural catheter for analgesia after total knee arthroplasty: the effect on physical rehabilitation and outcomes. J Arthroplasty 2006; 21: 1111-7.

[11] Hayek SM, Ritchey RM, Sessler D, et al. Continuous femoral nerve analgesia after unilateral total knee arthroplasty: stimulating versus nonstimulating catheters. Anesth Analg 2006; 103: 1565-70.

[12] Hebl JR, Dilger JA, Byer DE, et al. A pre-emptive multimodal pathway featuring peripheral nerve block improves perioperative outcomes after major orthopedic surgery. Reg Anesth Pain Med 2008; 33: 510-7.

[13] Buvanendran A, Kroin JS, Della Valle CJ, Kari M, Moric M, Tuman KJ. Perioperative oral pregabalin reduces chronic pain after total knee arthroplasty: a prospective, randomized, controlled trial. Anesth Analg 2010; 110: 199-207.

[14] Frassanito L, Vergari A, Zanghi F, Messina A, Bitondo M, Antonelli M. Post-operative analgesia following total knee arthroplasty: comparison of low-dose intrathecal morphine and single-shot ultrasound-guided femoral nerve block: a randomized, single blinded, controlled study. Eur Rev Med Pharmacol Sci 2010; 14: 589-96.

[15] Bowrey S, Hamer J, Bowler I, Symonds C, Hall JE. A comparison of 0.2 and $0.5 \mathrm{mg}$ intrathecal morphine for postoperative analgesia after total knee replacement. Anaesthesia 2005; 60: 449-52.

[16] Cole PJ, Craske DA, Wheatley RG. Efficacy and respiratory effects of low-dose spinal morphine for postoperative analgesia following knee arthroplasty. Br J Anaesth 2000; 85: 233-7.

[17] Patient Information: Anesthesia for Joint Replacement. Available at: http://anesthesia.stonybrook.edu/patient/orthope-dic/joint_replacement, [Accessed: Sept. $4^{\text {th }}$ 2012].

[18] Singh JA, Lewallen D. Predictors of pain and use of pain medications following primary Total Hip Arthroplasty (THA): 5,707 THAs at 2-years and 3,289 THAs at 5-years. BMC Musculoskelet Disord 2010; 11: 90.

[19] Ganapathy S, Wasserman RA, Watson JT, et al. Modified continuous femoral three-in-one block for postoperative pain after total knee arthroplasty. Anesth Analg 1999; 89: 1197-202.

[20] Brodner G, Buerkle H, Van Aken H, et al. Postoperative analgesia after knee surgery: a comparison of three different concentrations of ropivacaine for continuous femoral nerve blockade. Anesth Analg 2007; 105: 256-62.

[21] Barrington MJ, Olive DJ, McCutcheon CA, et al. Stimulating catheters for continuous femoral nerve blockade after total knee arthroplasty: a randomized, controlled, double-blinded trial. Anesth Analg 2008; 106: 1316-21.

[22] Wang AZ, Gu L, Zhou QH, Ni WZ, Jiang W. Ultrasound-guided continuous femoral nerve block for analgesia after total knee arthroplasty: catheter perpendicular to the nerve versus catheter parallel to the nerve. Reg Anesth Pain Med 2010; 35: 127-31.

[23] Wang H, Boctor B, Verner J. The effect of single-injection femoral nerve block on rehabilitation and length of hospital stay after total knee replacement. Reg Anesth Pain Med 2002; 27: 139-44.

[24] Gramke HF, de Rijke JM, van Kleef M, et al. Predictive factors of postoperative pain after day-case surgery. Clin J Pain 2009; 25: 455-60.

[25] Kalkman CJ, Visser K, Moen J, Bonsel GJ, Grobbee DE, Moons KG. Preoperative prediction of severe postoperative pain. Pain 2003; 105: 415-23

[26] Rosseland LA, Stubhaug A. Gender is a confounding factor in pain trials: women report more pain than men after arthroscopic surgery. Pain 2004; 112: 248-53.

[27] Faucett J, Gordon N, Levine J. Differences in postoperative pain severity among four ethnic groups. J Pain Symptom Manage 1994 9: 383-9.

[28] Banz VM, Christen B, Paul K, et al. Gender, age and ethnic aspects of analgesia in acute abdominal pain: is analgesia even across the groups? Intern Med J 2010; 42: 281-8

[29] Cancello R, Tounian A, Poitou C, Clément K. Adiposity signals, genetic and body weight regulation in humans. Diabetes Metab 2004; 30: 215-27.

[30] Lago R, Gómez R, Lago F, Gómez-Reino J, Gualillo O. Leptin beyond body weight regulation--current concepts concerning its role in immune function and inflammation. Cell Immunol 2008; 252: $139-45$.

[31] Guerre-Millo M. Adiponectin: an update. Diabetes Metab 2008; 34 : $12-8$

[32] Juge-Aubry CE, Henrichot E, Meier CA. Adipose tissue: regulator of inflammation. Best Pract Res Clin Endocrinol Metab 2005; 19: 547-66.

[33] Fantuzzi G. Adipose tissue, adipokines, and inflammation. J Allergy Clin Immunol 2005; 115: 911-9

[34] La Cava A, Matarese G. The weight of leptin in immunity. Nat Rev Immunol 2004; 4: 371-9.

[35] Vendrell J, Broch M, Vilarrasa N, et al. Resistin, adiponectin, ghrelin, leptin, and proinflammatory cytokines: relationships in obesity. Obes Res 2004; 12: 962-71.

[36] Considine RV, Sinha MK, Heiman ML, et al. Serum immunoreactive-leptin concentrations in normal-weight and obese humans. N Engl J Med 1996; 334: 292-5.

[37] Hickey MS, Israel RG, Gardiner SN, et al. Gender differences in serum leptin levels in humans. Biochem Mol Med 1996; 59: 1-6.

[38] Visser M, Bouter LM, McQuillan GM, Wener MH, Harris TB. Elevated C-reactive protein levels in overweight and obese adults. JAMA 1999; 282: 2131-5.

[39] Vgontzas AN, Papanicolaou DA, Bixler EO, Kales A, Tyson K, Chrousos GP. Elevation of plasma cytokines in disorders of excessive daytime sleepiness: role of sleep disturbance and obesity. J Clin Endocrinol Metab 1997; 82: 1313-6.

(C) Azim et al.; Licensee Bentham Open.

This is an open access article licensed under the terms of the Creative Commons Attribution Non-Commercial License (http:/creativecommons.org/licenses/by-nc/3.0/) which permits unrestricted, non-commercial use, distribution and reproduction in any medium, provided the work is properly cited. 